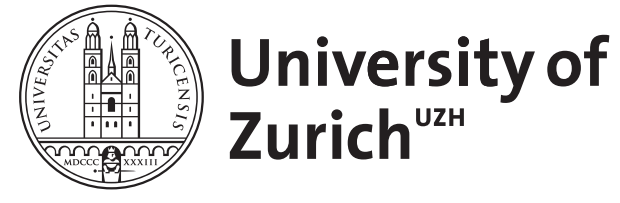

Zurich Open Repository and Archive

University of Zurich

University Library

Strickhofstrasse 39

CH-8057 Zurich

www.zora.uzh.ch

Year: 2013

\title{
Morbus Behçet
}

Weber, Nina ; Schaer, Dominik ; Vallelian, Florence

DOI: https://doi.org/10.1024/1661-8157/a001510

Posted at the Zurich Open Repository and Archive, University of Zurich ZORA URL: https://doi.org/10.5167/uzh-91587

Journal Article

Accepted Version

Originally published at:

Weber, Nina; Schaer, Dominik; Vallelian, Florence (2013). Morbus Behçet. Praxis, 102(24):1445-1453.

DOI: https://doi.org/10.1024/1661-8157/a001510 


\title{
Morbus Behçet
}

\author{
Definition/Leitsymptome:
}

Beim Morbus Behçet handelt es sich um ein inflammatorisches Syndrom, welches charakterisiert ist durch orale und genitale Ulcera, Entzündung verschiedener Augenabschnitte, vaskuläre und neurologische Begleiterkrankungen, Arthritis, renale Beteiligung sowie weitere systemische Manifestationen. Zentral in der Manifestation des Morbus Behcet ist eine Vaskulitis, welche die arterielle und/oder venöse Strombahn betreffen kann.

Die Patienten stellen sich primär oft aufgrund von rezidivierenden schmerzhaften mukokutanen und genitalen Aphten vor. Die weiteren Krankheitsmanifestationen sind vielfältig. Die grösste Morbidität und Mortalität ist aufgrund der okulären, vaskulären und neurologischen Manifestationen zu erwarten. Haut- und Gelenksbeteiligung sind häufig; renale Komplikationen sind im Vergleich zu anderen Vaskulitis-Syndromen selten.

Das charakteristische Krankheitsbild wurde erstmals 1937 durch den türkischen Arzt Hulusi Behçet beschrieben, nach dem die Erkrankung heute benannt ist. Der Morbus Behçet tritt gehäuft in der Region der ehemaligen Seidenstrasse auf, welche sich von Südostasien bis in die Mittelmeerregion erstreckt. Die höchste Prävalenz ist in der Türkei dokumentiert (80-370 Fälle auf 100'000 Pers.).

Typischerweise sind junge Erwachsene zwischen 20-40 Jahren betroffen; Männer und Frauen in gleicher Häufigkeit, wobei die Verläufe bei jungen Männern meistens deutlich schwerer sind. Die Krankheit tritt sporadisch auf, allerdings sind Familien mit gehäuftem Auftreten beschrieben.

\section{Im Artikel verwendete Abkürzungen}

ANCA Antineutrophile cytoplasmatische Antikörper

ANA Antinukleäre Antikörper

PR3 Proteinase-3-Anti-Neutrophilen-Cytoplasma-Antikörper

MPO Myeloperoxidase Antikörper

SLE Systemischer Lupus erythematodes 
Die klinischen Manifestationen des Morbus Behçet sind am ehesten auf eine Vaskulitis zurückzuführen, wobei Gefässe aller Grössen (kleine, mittelgrosse und grosse Gefässe) betroffen sein können. Generell sind sowohl die arterielle als auch die venöse Strombahn betroffen, wobei beim individuellen Patienten oft exklusiv venöse oder arterielle Vaskulitis-Manifestationen gefunden werden.

- Orale Ulcera: Bei fast allen Patienten mit Morbus Behçet kommt es zum Auftreten von mukokutanen Ulcera. Diese Aphten werden oft als sine-qua-non Manifestation der Krankheit betrachtet. Histologisch sind die Ulcera kaum von gewöhnlichen Aphten zu unterscheiden. Jedoch sind die Ulcera beim Morbus Behçet oft grösser, langsam heilend und oft sehr schmerzhaft. Sie sind typischerweise $2 \mathrm{~mm}-2 \mathrm{~cm}$ gross, mit scharfer Begrenzung und zeigen einen weissen bis gelblichen Grund.

Um die Definition eines Morbus Behçet zu erfüllen, sollten die Ulcera mindestens 3 Mal pro Jahr auftreten und nach jeweils 1-3 Wochen spontan abheilen.

- Urogenitale Ulcera: Bei ca. $75 \%$ der Patienten mit Morbus Behçet kommt es zum Auftreten von genitalen Aphten. Bei Männern zeigen sie sich oft am Skrotum, bei Frauen an der Vulva. Die Ulcera sind morphologisch ähnlich wie die oralen Aphten und ebenfalls sehr schmerzhaft Selten kann es auch zu Epidydimitis, Salpingitis oder Varikozelen kommen; eine Urethritis ist jedoch untypisch in diesem Symptomenkomplex.

- Vaskuläre Beteiligung: Wie bereits erwähnt sind wohl die meisten systemischen Manifestationen des Morbus Behçet durch eine Vaskulitis zu erklären. Die venöse Beteiligung ist insgesamt häufiger als die Arterielle. Typische Manifestationen sind oberflächliche und tiefe Venenthrombosen, Sinusvenenthrombosen, V. cava-Thrombosen und das Budd-ChiariSyndrom.

- Etwa ein Drittel der Patienten zeigen eine Vaskulitis der grossen Gefässe. Klinisch führt diese zu Blutungen, Aneurysmabildung, Thrombosen der Arterien und Venen und Varizenbildung. Am häufigsten sind Karotiden, Aorta, lliacal-, Femoral- und Poplitealgefässe betroffen. Intrazerebrale Gefässe oder Nierenarterien sind selten beteiligt. Typisch, wenn nicht gar pathognomonisch, ist die Beteiligung der Pulmonalarterien. Dabei kommt es zur Bildung von Pulmonalarterienaneurysmata, was klinisch zu Hämoptoe, Husten, Fieber, Dyspnoe und pleuritischen Schmerzen führen kann. Aneurysma der Pulmonalisarterien stellen wegen der hohen Blutungsgefahr eine Kontraindikation zur Antikoagulation dar und sollten bei Behçet Patienten mit Thrombosen aktiv gesucht werden.

Generell treten oberflächliche und tiefe Thrombosen gehäuft in einem Manifestations-Cluster mit anderen vaskulitischen Manifestationen (z.B. Aneurysmen) auf. Die Konstellation wird auch als Angio-Behçet bezeichnet.

Bei gleichzeitigem Auftreten von Aneurysmata der Pulmonalarterien und einer peripheren Thrombophlebitis spricht man vom Hughes-Stovin-Syndrom. Eine kardiale Beteiligung wurde bei weniger als $10 \%$ der Behçet-Patienten gefunden, betrifft aber meistens Männer mit arteriellen und venösen Läsionen.

- Kutane Manifestationen: Ein Hautbefall wird in rund $75 \%$ der Patienten gesehen. Die Hautmanifestationen sind vielfältig; es zeigen sich Akne-ähnliche pustulöse Läsionen an für Akne atypischen Hautregionen, Erythema nodosum, Pseudofollikulitis, oberflächliche Thrombophlebitiden, Pyoderma gangränosum oder Eryhthema nodosum ähnliche Läsionen oder eine palpable Purpura.

- Augenbeteiligung: Eine okuläre Mitbeteiligung ist insbesondere bei jungen Männern häufig und mit einer hohen Morbidität assoziiert. Da zu Beginn oft subklinisch muss eine Augenbeteiligung bei jedem Patienten mit Verdacht auf Morbus Behçet aktiv durch eine ophthalmologische Untersuchung gesucht werden. Eine beidseitige Panuveitis kann typischerweise auftreten. Diese tritt oft rezidivierend auf, wobei es zwischen den akuten Episoden meistens nicht zu einer kompletten Remission kommt.

Es gibt auch Fälle einer schweren vorderen Uveitis mit Hypopyon, einer retinalen Uveitis oder 
einer Optikusneuritis. Diese Manifestationen können mit einem irreversiblen Visusverlust einhergehen.

- Neurologische Mitbeteiligung: Weniger als $20 \%$ der Patienten zeigen eine neurologische Beteiligung, wobei ebenfalls Männer häufiger betroffen sind.

Auch hier zeigt sich eine grosse Spannbreite der klinischen Symptome. Es kann zu fokalen parenchymalen Läsionen im ZNS kommen. Weiter zeigen sich aseptische Meningitiden, epileptische Anfälle oder eine Encephalitis, welche im Verlauf in

Persönlichkeitsveränderungen oder Demenz münden können. Eine periphere Polyneuropathie ist hingegen untypisch.

- Arthritis: Ca. 50\% der Patienten zeigen eine Gelenksbeteiligung. Dabei tritt typischerweise eine nicht-erosive, asymmetrische, nicht-deformierende Arthritis der mittelgrossen und grossen Gelenke auf.

- Gastrointestinale Beteiligung: viele Patienten leiden an rezidivierenden Bauchschmerzen. Ulzerationen im Dünn- und Dickdarm können bei wenigen Patienten nachgewiesen werden. Eine Behçet-Krankheit mit intestinalen Ulzerationen ist unter Umständen schwierig von einem Morbus Crohn oder einer Colitis ulcerosa mit extraintestinalen Manifestationen zu unterschieden.

- Nierenbeteiligung: Im Vergleich zu anderen Vaskulitiden ist die renale Beteiligung beim Morbus Behçet seltener und meist auch weniger schwerwiegend. Klinisch bzw. im Labor zeigt sich eine Proteinurie, Hämaturie, leichtgradige Niereninsuffizienz; selten ist die

Niereninsuffizienz fortschreitend. Die zu Grunde liegende Pathogenese ist oftmals eine sekundäre Amyloidose, eine Glomerulonephritis, renale Aneurysmata oder eine interstitielle Nephritis.

- Allgemeine Symptome wie Fatigue und B-Symptomatik werden häufig bei Behçet-Patienten beobachtet. 


\section{Routinelabor}

Es gibt keine pathognomonischen Laborveränderungen für den Morbus Behçet. Da es sich um einen entzündlichen Prozess handelt, kann man neben der typischen Klinik meistens eine beschleunigte Blutsenkungsreaktion (BSR) und ein erhöhtes C-reaktives Protein (CRP) finden. Allerdings können BSR und CRP trotz akuter Krankheit normal sein.

\section{Erweitertes Labor}

Folgende Laborparameter geben Auskunft über die betroffenen Organsysteme und sollten im Rahmen der Erstabklärungen, insbesondere zum Ausschluss anderer Ursachen bestimmt werden:

- Serum-Kreatinin und Urinstatus mit Sediment mit Frage nach Zylindern und dysmorphen Erythrozyten. Diese Untersuchungen ermöglichen, Hinweise auf eine Vaskulitis mit Nierenbeteiligung zu finden.

- Positive ANA und anti-DNS können hinweisend auf einen SLE sein

- C3 und C4: erniedrigte Werte bei SLE, Kryoglobulinämie oder anderen Immunkomplex vermittelten Vaskulitiden

- c-ANCA (Zielantigen: Proteinase 3): stark hinweisend auf Granulomatose mit Polyangiitis oder Eosinophile Granulomatose mit Polyangiitis

p-ANCA (Zielantigene: Myeloperoxidase, Elastase, Kathepsin G) bei mikroskopischer Polyangiitis. p-ANCA sind weniger spezifisch für das Vorliegen einer Vaskulitis als c-ANCA

- Serologien für Hepatitis B, C, CMV, EBV, HIV, Parvovirus B19

- HLA-B51 Expression : trotz klarer Assoziation ist der Test weder sensitiv noch spezifisch und wird nicht routinemässig empfohlen

\section{Weitere Abklärungen}

- Eine ophthalmologische Untersuchung sollte bei jedem Verdachtsfall erfolgen

- Pathergie-Test : Das Pathergiephänomen, wie es sich im Pathergie-Test zeigt, ist eine Besonderheit des Morbus Behçet und beschreibt die Hyperreaktivität der Haut am Ort eines Nadeleinstichs Das Auftreten einer Papel oder Pustel nach nach Injektion von $0.5 \mathrm{ml}$ physiologischer Kochsalzlösung intradermal wird als positiver Pathergie-Test interpretiert. Der Test ist sehr spezifisch für einen Morbus Behcet, allerdings insbesondere in der zentraleuropäischen Bevölkerung wenig sensitiv.

- Je nach Klinik EKG, Thorax röntgen, Lungenfunktionsprüfung, Liquorpunktion und Bildgebung des ZNS

- MR- oder CT-Angiographie bei Verdacht auf Vaskulitis der mittelgrossen oder grossen Gefässe. Suche nach Aneurysmata, Gefässverschlüssen oder Wandirregularitäten.

- Das PET-CT kann Hinweise für Grossgefäss-Vaskulitis und inflammatorische Thrombosen liefern.

- $\quad$ MR bei Verdacht auf ZNS Beteiligung

- Endoskopie bei Verdacht auf intestinale Beteiligung 


\section{Diagnosekriterien}

Der Morbus Behçet kann nicht anhand von typischen Laborbefunden diagnostiziert werden. Die Diagnose ist daher anhand der klinischen Manifestationen zu stellen. Ein inkomplettes, therapiebedürftiges Behcet-Syndrom kann gelegentlich auch dann vorliegen sein wenn die publizierten Diagnosekriterien nicht vollständig erfüllt sind.

Tab. 1 Diagnosekriterien gemäss den internationalen Kriterien für Morbus Behçet (ICBD) von 2006. Erforderlich sind mindestens 3/8 Punkten

- Genitale Aphten (2 Punkte)

- Okulärer Befall (Anteriore oder posteriore Uveitis, retinale Vaskulitis) (2 Punkte)

- Orale Aphten (1 Punkt)

- Hautmanifestationen (Pseudofollikulitis, Erythema nodosum) (1 Punkt)

- Vaskuläre Manifestationen (Oberflächliche Phlebitis, tiefe Venenthrombose, Thrombose einer grossen Vene, arterielle Thrombose, Aneurysma) (1 Punkt)

- Positiver Pathergie-Test (1 Punkt)

\section{Differentialdiagnose}

Die möglichen Differentialdiagnosen sind sehr breit und ergeben sich aus der klinischen Präsentation.

Tab. 2 Differentialdiagnose der oralen Ulzera:

- Benigne orale Aphten

- Herpes simplex, HIV

- Pemphigus, Pemphigoid, Steven-Johnson Syndrome

- Autoentzündliche Syndrome

- Morbus Crohn

- SLE

Tab.2 Differentialdiagnose der Behçet-Krankheit:

Formatiert: Deutsch (Schweiz)

\begin{tabular}{|l|l|l|}
\hline $\begin{array}{l}\text { Tab.3 Differentialdiagnose der } \\
\text { Behçet-Krankheit: }\end{array}$ & Überlappende Symptome & Unterscheidungskriterien \\
\hline SLE & $\begin{array}{l}\text { Orale Ulcera, nicht-erosive } \\
\text { Arthritis, neurologische und } \\
\text { vaskuläre Symptome }\end{array}$ & ANA, C3/4 erniedrigt bei SLE \\
\hline $\begin{array}{l}\text { HLA-B27 pos. } \\
\text { Spondylarthritiden }\end{array}$ & $\begin{array}{l}\text { Orale Ulcera, Uveitis, Arthritis, } \\
\text { Haut- und gastrointestinale } \\
\text { Beteiligung }\end{array}$ & HLA-B27-Positivität \\
\hline M. Crohn & $\begin{array}{l}\text { Orale Ulcera, nicht-erosive } \\
\text { Arthritis, Augen-, Haut- und } \\
\text { gastrointestinale Beteiligung }\end{array}$ & $\begin{array}{l}\text { Histologisch: Granulome } \\
\text { Skleritis }\end{array}$ \\
\hline Colitis ulcerosa & $\begin{array}{l}\text { Orale Ulcera, nicht-erosive } \\
\text { Arthritis, Uveitis, Haut- und } \\
\text { gastrointestinale Beschwerden }\end{array}$ & $\begin{array}{l}\text { Backwash-Ileitis } \\
\text { Histologisch: Kryptenabszesse }\end{array}$ \\
\hline Eutoinflammatorische & $\begin{array}{l}\text { Urogenitale Ulcera, } \\
\text { Hautmanifestation, nicht- } \\
\text { erosive Arthritis, neurologische } \\
\text { Beteiligung }\end{array}$ & $\begin{array}{l}\text { Fieberschübe, } \\
\text { Krankheitsbeginn in der } \\
\text { Abklärungen }\end{array}$ \\
\hline Sarkoidose & $\begin{array}{l}\text { Orale Ulcera, } \\
\text { Lungenbeteiligung, Erythema }\end{array}$ & Histologisch: Granulome \\
\hline
\end{tabular}

Formatiert: Deutsch (Schweiz) 


\begin{tabular}{|l|l|l|}
\hline & $\begin{array}{l}\text { nodosum, neurologische und } \\
\text { Hautmanifestationen }\end{array}$ & \\
\hline Zyklische Neutropenie & Orale Ulcera & $\begin{array}{l}\text { Zyklischer Verlauf der } \\
\text { Neutropenie }\end{array}$ \\
\hline SAPHO-Syndrom & $\begin{array}{l}\text { Arthritis, Akne, pustuläre } \\
\text { Läsionen }\end{array}$ & Osteitis, Hyperostosis \\
\hline ANCA-pos. Vaskulitis & $\begin{array}{l}\text { Arthritis, Augen- und } \\
\text { Hautmanifestationen }\end{array}$ & ANCA \\
\hline Sonstiges & $\begin{array}{l}\text { Multiple Sklerose, Tuberkulose, } \\
\text { HIV, Malignome }\end{array}$ & \\
\hline
\end{tabular}

Einteilung der Vaskulitiden gemäss der internationalen Chapel Hill Consensus Conference 2012 (CHCC). Nomenklatur nach Grösse der betroffenen Gefässe eingeteilt.

\begin{tabular}{|c|c|}
\hline Vaskulitis der grossen Gefässe & $\begin{array}{ll}\text { - } & \text { Takayasu-Arteriitis } \\
\text { - } & \text { Riesenzell-Arteriitis }\end{array}$ \\
\hline Vaskulitis der mittelgrossen Gefässe & $\begin{array}{ll}\text { - } & \text { Panarteriitis nodosa } \\
\text { - } & \text { Kawasaki-Krankheit } \\
\text { - } & \text { Primäre Angiitis des ZNS }\end{array}$ \\
\hline Vaskulitis der kleinen Gefässe & $\begin{array}{ll}\text { - } & \text { Eosinophile Granulomatose mit } \\
& \text { Polyangiitis (ehemals Churg-Strauss) } \\
\text { - } & \text { Granulomatose mit Polyangiitis (ehemals } \\
& \text { Morbus Wegener) } \\
\text { - } & \text { Mikroskopische Polyangiitis } \\
\text { - } & \text { IgA-Vaskulitis (Purpura Schönlein- } \\
& \text { Henoch) } \\
\text { - } \quad \text { Kryoglobulinämie } \\
\text { - } \quad \text { Hypersensitivitätsvaskulitis } \\
\text { - Sekundäre Vaskulitis i.R. einer } \\
\text { Systemerkrankung wie z.B. M. Behçet, } \\
\text { - SLE, rheumatoide Arthritis } \\
\text { Sekundäre Vaskulitis nach viralen } \\
\text { Infekten, z.B. nach Hepatitis B, C, HIV, } \\
\text { CMV, EBV, Parvovirus B19 }\end{array}$ \\
\hline
\end{tabular}


Analog zu anderen primären Entzündungskrankheiten steht eine anti-inflammatorische und/oder immunsuppressive Therapie im Vordergrund, wobei beim Morbus Behçet in Abhängigkeit vom Organbefall und dem Schweregrad der Erkrankung verschiedene spezifische Therapieempfehlungen zu beachten sind.

Wenn die mukokutanen Symptome vordergründig sind, haben sich neben der topischen Steroidtherapie gute Resultate mit Colchicin gezeigt. Aufgrund der gastrointestinalen Nebenwirkungen sollten Dosen von $>1.5 \mathrm{mg}$ pro Tag vermieden werden. Bei fehlender Besserung unter Colchicin, muss eine Therapie mit Azathioprin in Erwägung gezogen werden. Die initiale Imurek-Dosis ist üblicherweise $1 \mathrm{mg} / \mathrm{kg} / \mathrm{d}$. und kann nach 3-4 Wochen stufenweise gesteigert werden bis max. $2.5 \mathrm{mg} / \mathrm{kg} / \mathrm{d}$.

Bei jeder immunsuppressiven Therapie müssen die Substanz-spezifischen Empfehlungen bezüglich Monitoring (z.B. Blutbild, Leberwerte), erforderlichen Basisuntersuchungen (z.B. Screening für latente Tuberkulose und Hepatitis) sowie Patientenberatung (z.B. bezüglich Kontrazeption, teratogenem Risiko) beachtet werden. In der Schweiz stellt die Schweizerische Gesellschaft für Rheumatologie auf ihrer Webseite Empfehlungen für die häufig eingesetzten Immunsuppressive zur Verfügung (http://www.rheuma-net.ch).

Uveitiden werden neben der lokalen Therapie durch den Ophthalmologen oft erfolgreich mit Azathioprin behandelt. Bei Unverträglichkeit oder Therapieresistenz, sind Immunsuppressiva wie Mycophenolat oder Cyclosporin zu empfehlen. Anti-TNF-Therapien werden zunehmend bei refraktären Verläufen eingesetzt.

Der Neurobehçet mit parenchymatösen Läsionen ist oft schwierig zu kontrollieren. In der akuten Phase werden hochdosiert Glucocorticoide eingesetzt, Azathioprin, Cyclophosphamid und TNFBlocker stehen als steroidsparende Medikamente für Induktionstherapie zur Verfügung. Für vaskulitische Manifestationen der grossen Gefässe wird nach wie vor in erster Linie Cyclophosphamid in Kombination mit Glucocorticoiden eingesetzt.

In den letzten Jahren hat der Stellenwert der Biologika als Therapie der Vaskulitiden insgesamt und speziell auch des Morbus Behcet zugenommen. Die breiteste publizierte Erfahrung besteht für den TNF-Blocker Infliximab, andere TNF-Blocker wie Adalimumab scheinen jedoch auch wirksam zu sein. Tocilizumab, ein IL-6-Rezeptor Antikörper hat erfolgversprechende Ergebnisse bei einzelnen Fallberichten erzielt und sollte bei refraktären Verläufen in Betracht gezogen werden.

Der Stellungswert der Antikoagulation bei Thrombosen ist umstritten. Die Zurückhaltung gewisser Experten bezieht sich auch das geringe Embolie-Risiko der inflammatorischen Thromben und die Tatsache, dass das Thromboserisiko beim Morbus Behçet wahrscheinlich weitaus stärker von einer optimalen Entzündungskontrolle als von einer Suppression der plasmatischen Gerinnungsaktivität abhängig ist. Zudem kann das Blutungsrisiko bei Patienten mit Aneurysmata (z.B.

Pulmonalisaneurysmata) deutlich erhöht sein und eine Kontraindikation zur Antikoagulation darstellen. In unserem Erfahrungskreis werden allerdings die meisten Behçet Patienten mit Thrombosen antikoaguliert, wobei potentieller Nutzen und Risiken bei jedem Patienten anhand seiner Krankheitsmanifestationen und Komorbiditäten diskutiert werden müssen.

\section{Prognose:}

Die Prognose der Behçet Patienten hat sich über den letzten Jahren deutlich verbessert und die Mehrheit der Patienten können in eine Remission gebracht. Allerdings bleibt die Morbidität der Erkrankung, insbesondere bei Augen- und/oder ZNS-Befall beträchtlich.

Die Mortalität ist mit 3-4\% gering und auf primär den Befall des Herzens und der grossen Gefässe (insbesondere akute Blutungen bei Pulmonalisaneurysmata) zurückzuführen. Junge Männer haben generell eine schlechtere Prognose, die Krankheitsaktivität nimmt in der Regel mit zunehmendem Alter ab. 


\section{Fallbericht eines 30-jährigen Patienten}

प

$\mathrm{JL}$ : Vorstellung eines 30 -jährigen Patienten aufgrund von seit 3 Wochen bestehenden rechtsseitigen Kopfschmerzen und allgemeinem Krankheitsgefühl. Kein Ansprechen auf Analgetika.

Zusätzlich akne-ähnliche Veränderungen im Dekolleté-Bereich sowie seit längerem rezidivierende sehr schmerzhafte orale, einmalig auch genitale Aphten.

SA: Gewichtsverlust 2-3kg in 3 Wochen, vermehrtes Schwitzen.

PA: blande

Medikamente: Dafalgan, Ibuprofen
Weg zur Diagnose/Kommentar Hinweisend auf eine Systemerkrankung sind in diesem Fall die Allgemeinsymptome (Gewichtsverlust, Müdigkeit, allg. Krankheitsgefühl, Schwitzen) in Kombination mit den Haut- und Schleimhautbefunden. Bei zusätzlich vorliegenden Kopfschmerzen könnte auch das ZNS im weitesten Sinne mitbeteiligt sein.

\section{d}

Status

30-jähriger Patient in leicht reduziertem AZ und schlankem EZ. Afebril, kreislaufstabil.

Papulopustulöse Effloreszenzen im Dekolleté-Bereich, orale Ulcera, aktuell keine genitalen Ulcera,

Druckdolenz temporal rechts.

Ansonsten unauffälliger Status.

Routinelabor

CRP 142mg/L ( $\uparrow \uparrow)$, BSR $76 \mathrm{~mm} / \mathrm{h}(\uparrow \uparrow)$, Leukozyten

$15.2 \mathrm{G} / \mathrm{L}(\uparrow \uparrow \uparrow)$, Kreatinin $65 \mu \mathrm{mol} / \mathrm{L}$, Urinstatus unauffällig

Weitere Befunde

Im MR-Angio Nachweis einer Thrombose im Sinus transversus rechts, welche sich bis in die V. jugularis fortzieht.

\section{Erweitertes Labor}

ANA, ANCA negativ, negative Serologien für Hepatitis B, C, EBV, CMV, HIV, Parvovirus B19

Aktuelle Therapie

Hochdosiert Steroide und Beginn einer oralen

Antikoagulation.

\section{Weg zur Diagnose/Kommentar}

Routinelabor (Entzündungsparameter, Kreatinin, U-Status, Leberparameter) MR-Angio des ZNS

Durchführung eines Pathergie-Tests
Die Diagnose eines $\mathrm{M}$. Behçet kann gemäss den internationalen Kriterien (ICBD) gestellt werden bei erfüllten 6/8 Punkten (mind. 3 Pkt. erforderlich):

- St.n. genitalen Ulcera (2 Pkt.)

- Hautbefund (Pseudofolikullitis) (1 Pkt.)

- Orale Ulcera (1 Pkt.)

- Vaskuläre Befunde

(Sinusvenenthrombose) (1 Pkt.)

- Pos. Pathergie-Test (1 Pkt.) 
Azathioprin. Vor Beginn mit Azathioprin wurde eine chronische Hepatitis B ausgeschlossen.

Bei voraussichtlich längerer Gabe von Steroiden wurde auch Calcium und Vitamin D substituiert sowie eine Knochendichtmessung durchgeführt.

Im längerfristigen Verlauf Normalisierung der Entzündungsparameter und deutliche Besserung der klinischen Beschwerden. 


\section{Fragen zum Morbus Behçet}

\section{Frage 1}

Welche Symptome lassen typischerweise an einen Morbus Behçet denken? (Mehrfachauswahl, mehrere richtige Antworten)

a) Rezidivierend schmerzhafte orale und genitale Ulcera

b) Akutes Nierenversagen

c) Symmetrische Arthritis der MCP und PIP-Gelenke

d) Pseudofollikulitis und ein positiver Pathergie-Test

e) Husten, Hämoptoe, Dyspnoe, radiologisch Nachweis von Pulmonalarterien-Aneurysmata

\section{Frage 2}

Die Diagnose eines Morbus Behcet kann durch einen positiven Pathergie-Test gestellt werden weil der Pathergie-Test spezifisch für den Morbus Behçet ist.

(Einfache Auswahl, 1 richtige Antwort)

a) 1.richtig, 2.falsch

b) 1.falsch, 2.richtig

c) alles richtig

d) alles falsch

e)1.richtig, 2.richtig, „weil“ falsch

\section{Frage 3}

Ophthalmologische Untersuchungen sollten bei allen Patienten mit Morbus Behçet erfolgen weil

Uveitiden asymptomatisch sein können

(Einfache Auswahl, 1 richtige Antwort)

a) 1.richtig, 2.falsch

b) 1. falsch, 2.richtig

c) alles richtig

d) alles falsch

e)1.richtig, 2.richtig, „weil“ falsch

\section{Frage 4}

Welche Untersuchungen sind sinnvoll zur Abklärung eines Morbus Behçet? (Mehrfachauswahl, mehrere richtige Antworten)

a) Die Diagnose kann leicht durch Bestimmung spezifischer auto-Antikörper gestellt werden

b) Pathergie-Test

c) ANA, ANCA, C3/4, Hepatitis-, EBV-, CMV-, Parvovirus B19-Serologie, HIV

d) MR-Angio Schädel

e) Ophthalmologische Untersuchung 


\section{Frage 5}

Was sind mögliche Behandlungsansätze beim Morbus Behçet?

(Mehrfachauswahl, mehrere richtige Antworten)

a) Topische Steroide oder Colchicin zur Behandlung von mukokutanen Ulcera

b) Bei Uveitiden kann Azathioprin eingesetzt werden

c) Bei einem Neuro-Behçet muss die Gabe von Prednison vermieden werden

d) Primär sollten auslösende Infektionen behandelt werden, eine weiterführende Therapie ist nicht sinnvoll

e) Bei nachgewiesenen Thrombosen sollte eine Antikoagulation als Monotherapie erfolgen

\section{Autoren}

Dr. med. Nina Weber, PD Dr. med. Dominik Schaer, Dr. med. Florence Vallelian, Klinik und Poliklinik für Innere Medizin, Sprechstunde für systemische Entzündungskrankheiten, Universitätsspital Zürich

\section{Korrespondenzadresse}

Formatiert: Deutsch (Schweiz)

Dr. med. Florence Vallelian

Klinik und Poliklinik für Innere Medizin

Sprechstunde für systemische Entzündungskrankheiten

Rämistrasse 100

8091 Zürich

florence.vallelian@usz.ch

\section{Bibliographie}

1. Nicola L. Ambrosa and Dorian O. Haskard. Differential diagnosis and management of Behçet syndrome. Nature Reviews Rheumatology, Vol. 9, Febr. 2013

2. Gulen Hatemi et al. Behcet's Syndrome. Rheum Dis Clin N Am 39 (2013) 245-261.

Formatiert: Englisch (USA)

3. Aguiar de Sousa D, Mestre T, Ferro JM. Cerebral venous thrombosis in Behçet's disease: a systematic review. J Neurol 2011;258(5):719-27.

Formatiert: Englisch (USA)

4. Saadoun D, Wechsler B, Resche-Rigon M, et al. Cerebral venous thrombosis in

Behcet's disease. Arthritis Rheum 2009;61(4):518-26.

Formatiert: Englisch (USA)

5. Böcker, Denk, Heinz, Moch. Pathologie, 3. Auflage. Urban \& Fischer Verlag/Elsevier GmbH; (Auflage: 3. 2004) 


\section{Antworten zu den Fragen zu XY aus PRAXIS Nr.}

\section{Frage 1}

\section{Richtig ist Antwort a/d/e}

$\mathrm{Ad} \mathrm{a} / \mathrm{d} / \mathrm{e}$ ) Orale und genitale Ulcerationen, Hautmanifestationen wie Pseudofollikulitis sowie der positive Pathergietest als auch die Pulmonalarterienaneurysmata sind Teil der internationalen Diagnosekriterien (ICBD) für den Morbus Behçet.

Ad b) Ein milder renaler Befall ist möglich, dies kann sich manifestieren mit einer Proteinurie, Hämaturie sowie einer geringen Niereninsuffizienz. Ein Fortschreiten zum terminalen Nierenversagen ist untypisch in diesem Krankheitsbild.

Ad c) Bei ca. 50\% der Patienten tritt eine Gelenksbeteiligung auf; diese ist jedoch meist asymmetrisch und betrifft die mittelgrossen und grossen Gelenke.

\section{Frage 2}

\section{Richtig ist Antwort b}

Ein positiver Pathergie-Test reicht nicht um die Diagnose stellen zu können. Gemäss den internationalen Kriterien für Morbus Behçet sind mindestens 4/8 Punkte erforderlich. Der PathergieTest ist zwar ein wenig sensitiver Test aber im entsprechenden klinischen Kontext spezifisch für den Morbus Behçet.

\section{Frage 3}

\section{Richtig ist Antwort c}

Eine okuläre Mitbeteiligung ist häufig. Da diese auch subklinisch verlaufen kann und mit einer hohen Morbidität verbunden ist, sollte eine ophthalmologische Untersuchung bei allen Patienten mit Verdacht auf Morbus Behçet erfolgen.

\section{Frage 4}

\section{Richtig ist Antwort b/c/d/e}

Ad a) Es sind keine spezifischen Antikörper zur Diagnose eines Morbus Behçet bekannt. Die Diagnose muss aufgrund der internationalen Diagnosekriterien gestellt werden. Im Labor sind oft unspezifisch erhöhte Entzündungsparameter zu sehen.

Ad b) Ein positiver Pathergie-Test ist Teil der internationalen Diagnosekriterien.

Ad c) Zum Ausschluss einer anderen zugrundeliegenden Vaskulitis sollten diese Antikörper bestimmt werden. Zudem sollte ein viraler Infekt (HIV, Hep B/C, CMV, EBV, Parvovirus B19; je nach Klinik auch weitere) ausgeschlossen werden.

Ad d) Bei Kopfschmerzen muss eine Bildgebung des Kopfes erfolgen. Hier sollte nach Thrombosen, Aneurysmata oder sonstigen Gefässwandirregularitäten gesucht werden. Bei neurologischen Auffälligkeiten muss auch an einen Neuro-Behçet gedacht werden. Dabei können sich im MR fokale Parenchymläsionen zeigen. Der Liquorbefund einer aseptischen Meningitis unterstützt den Verdacht auf einen ZNS-Befall.

Ad e) Die okuläre Beteiligung ist gefürchtet und kann sich als oft bilaterale Panuveitis, retinale Uveitis oder Optikusneuritis manifestieren.

\section{Frage 5}

\section{Richtig ist Antwort a/b}

Ad a) Mit topischen Steroiden sowie Colchicin können mukokutane Symptome gut behandelt werden $\mathrm{Ad}$ b) Uveitiden werden meistens erfolgreich mit Azathioprin behandelt. Bei Unverträglichkeit, sind Immunsuppressiva wie Mycophenolat oder Cyclosporin zu empfehlen. Anti-TNF-Therapien werden zunehmend bei refraktären Verläufen eingesetzt.

Ad c) Bei Neuro-Behçet sind initial hochdosierte Glucocorticoide die Therapie der Wahl; als steroidsparende Medikation wird im Verlauf primär Azathioprin empfohlen.

Ad d/e) Grundsätzlich sollte immer eine immunsuppressive/anti-inflammatorische Therapie erfolgen. Die Antikoagulation als Monotherapie therapiert nicht die Grundkrankheit und ist demzufolge nicht ausreichend. 


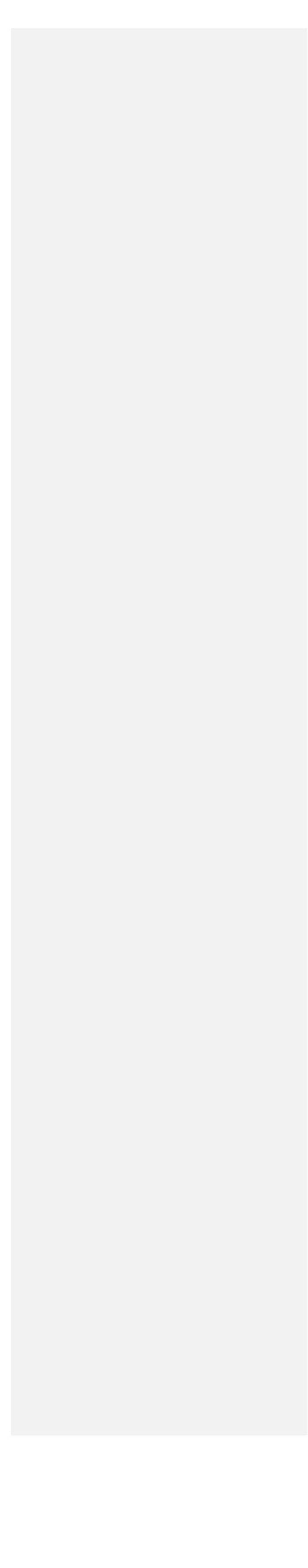

